many social determinants of health. NEs can be conceptualised as differing from other research in three important ways. First, unlike trials, investigators cannot allocate the exposure of interest which is instead externally decided. Second, researchers are unable to influence the exposure's characteristics which results in uncertainty of the fidelity, dose and whether the NE will occur at all. Third, and in contrast to many observational studies, the timing of the exposure and hence research itself, cannot be chosen by researchers.

Methods Using three case studies of research on H1N1 (two quantitative sero-epidemiology studies, one qualitative study) we identified key factors that allowed us to successfully conduct our investigation of a NE. We compared these factors with the wider NE literature to identify common barriers and facilitators to research. Results We identified key external factors that influence capacity to successfully take advantage of NEs. As a result of the uncertainty of a NE occurring and the rapid response required, flexibility is needed by researchers, policymakers, practitioners, ethics committees and funders. This is achievable when research is perceived as immediately important for health (as in H1N1) but may otherwise be difficult.

Discussion We suggest researchers investigating NEs face additional challenges to traditional observational studies. While some barriers are insurmountable, actions such as rapid response funding, fasttrack ethics procedures and improvements in routine data can create a more conducive environment allowing policy-relevant evaluation

\section{P1-187 ACCESS TO ALCOHOL OUTLETS AND HARMFUL ALCOHOL CONSUMPTION: A MULTILEVEL STUDY IN MELBOURNE, AUSTRALIA}

doi:10.1136/jech.2011.142976d.80

${ }^{1} \mathrm{~A}$ Kavanagh, ${ }^{2} \mathrm{M}$ Kelly, ${ }^{1} \mathrm{~L}$ Krnjacki, ${ }^{3} \mathrm{~L}$ Thornton, ${ }^{4} \mathrm{D}$ Jolley, ${ }^{5} \mathrm{~S} \vee$ Subramanian, ${ }^{6} \mathrm{G}$ Turrell, ${ }^{1} \mathrm{R}$ Bentley. ${ }^{1}$ The University of Melbourne, Victoria, Australia; ${ }^{2}$ La Trobe University, Victoria, Australia; ${ }^{3}$ Deakin University, Victoria, Australia; ${ }^{4}$ Monash University, Victoria, Australia; ${ }^{5}$ Harvard University, Boston, USA; ${ }^{6}$ Oueensland University of Technology, Queensland, Australia

Introduction While developed countries have either introduced, or are considering, legislation to restrict the number of alcohol outlets, there is little evidence to support this strategy except in the USA.

Methods Multilevel study of 2334 adults aged 18 to 75 years from 49 census collector districts (the smallest spatial unit in Australia at the time of survey) in Melbourne, Australia. Alcohol outlet density was defined as the number of outlets within a one kilometre road network of respondents' homes and proximity was the shortest road network distance to the closest outlet from their home. Using multilevel logistic regression we estimated the association between outlet density and proximity and four measures of harmful alcohol consumption: drinking at levels associated short-term harm at least weekly and monthly; drinking at levels associated with long-term harm and frequency of consumption.

Results Density of outlets was associated with increased risk of alcohol related harm with the strongest association evident for drinking at levels associated with short term harm at least weekly (OR 1.10, 95\% CI 1.04 to 1.16). When density was fitted as a categorical variable, the highest risk of drinking at levels associated with short-term harm was when there were eight or more outlets (short-term harm weekly: OR 2.36, 95\% CI 1.22 to 4.54 and shortterm harm monthly: OR $1.80,95 \%$ CI 1.07 to 3.04$)$. We found no evidence to support an association between proximity and harmful alcohol consumption.

Conclusion Restricting the number of off-premise alcohol outlets is likely to reduce levels of harmful alcohol consumption.

\section{P1-188 ANALYSIS OF QUESTIONNAIRE SURVEYS ON MOTHERS' ANXIETIES ABOUT CHILD-REARING IN RURAL AREAS OF JAPAN}

doi:10.1136/jech.2011.142976d.81

${ }^{1} \mathrm{H}$ Kawasaki, ${ }^{2} \mathrm{M}$ Nlshiyama, ${ }^{3} \mathrm{M}$ Fujita, ${ }^{3} \mathrm{P}$ D'Angelo, ${ }^{1} \mathrm{~S}$ Moriwaki. ${ }^{1}$ Hiroshima University, Hiroshima, Japan; 'Hiroshima Bunkyo Women's University, Hiroshima, Japan; ${ }^{3}$ Doi Clinic, Hiroshima, Japan

Background In Japan, as society changes, becoming ever more isolated and with rises in nuclear families, mothers are increasingly concerned and uneasy about child care. These changes have also coincided with a significant decrease in birth rates. The government took out the "indicator of support plan for the 21st century" in 2001. So, each prefecture had to draw up a concrete plan by 2005 .

Purpose The purpose of study was to explore factors affecting mothers' anxieties and to evaluate effects of a child care support plan. Sample and Statistical Analysis The questionnaire surveys were conducted with 1000 mothers, selected randomly, in 2002 and 2008. Mothers' anxiety was measured using feelings of loneliness, confusion about child-rearing and lack of standards as the children's development process. These were analysed using "ordered categorical logistic regression". The explanatory variables were mother's age, child's age and child-rearing methods.

Results 'Feelings of loneliness' was negatively correlated with a child's age ( $p$ value $=0.07$ ) and a maternal age $(p$ value $=0.044)$. Mothers' mental instability and a lack of families' cooperation significantly related with 'feelings of loneliness' ( $p$ values $=0.000$, 0.000 , respectively). "Feelings of loneliness" and "confusion about child-rearing" in 2008 (after implementation of the child care support plan) were reduced compared with those in 2002 ( $p$ values $=0.069,0.065$, respectively).

Conclusions The introduction of a child care support plan including opportunities for counselling, friendship and activities increased family co-operation and more social interaction for both mother and child. The research shows these can be effective methods in reducing mothers' anxieties.

\section{P1-189 NET BENEFIT FRACTION AS A MODIFIED POPULATION ATTRIBUTABLE FRACTION TO EVALUATE THE CLINICAL PERFORMANCE OF A RISK FUNCTION}

doi:10.1136/jech.2011.142976d.82

${ }^{1,2} \mathrm{D}$ Khalili, ${ }^{*}{ }^{1} Y$ Mehrabi, ${ }^{2} \mathrm{M}$ Bozorgmanesh. ${ }^{1}$ Department of Epidemiology, School of public health, Shahid Beheshti University of Medical Sciences, Tehran, Iran; ${ }^{2}$ Prevention of Metabolic Disorders Research Center, Research Institute for Endocrine Sciences, Shahid Beheshti University of Medical Sciences, Tehran, Iran

Introduction Net benefit (NB) has been suggested to verify the clinical usefulness of a model beyond its discrimination and calibration but it has a complex meaning in prediction models. Here we define net benefit fraction (NBF) using Population Attributable Fraction (PAF) index.

Methods According to Vickers and Elkin (2006), in models that predict diseases to treat high risk individuals, NB is defined as true positive (TP) rate minus weighted false positive (wFP) rate; the weight is the odds at the threshold probability for treatment $(\mathrm{Pt} /[1$ $\mathrm{Pt}]$ ). Dividing NB by its maximum, incidence of disease, gives the portion of incidence prevented by the treatment. A shortcoming of $\mathrm{NB}$ is that it assumes the treatment reduces the incidence in TPs to zero. On the other hand, PAF is defined as reduction of the risk to below the threshold, that is, to that of low risk population. However, PAF does not take into account the false positives. Therefore, we suggest NBF calculated as PAF minus wFP rate, the latter divided by incidence. We applied the method to calculate the clinical performance of Framingham risk function at routine 